\title{
Particle Swarm Optimization for Antenna Port Placement in Coordinated Multi-Point Systems
}

\author{
J. C. M. Feitosa, T. F. Maciel, E. M. G. Stancanelli, W. C. Freitas Jr., and F. R. P. Cavalcanti
}

\begin{abstract}
Coordinated Multi-Point (CoMP), also termed Distributed Antenna System (DAS), has emerged in recent years in the $3^{\text {rd }}$ Generation Partnership Project (3GPP) Long Term Evolution (LTE) as a promising architecture to enhance the link performance and, consequently, the capacity of wireless communication systems. A CoMP system consists of several Antenna Ports (APs) geographically spread over a coverage area and connected through a fast backhaul to an Enhanced Node $B$ (eNB). The optimal placement of a number of distributed APs depends, e.g., on the distribution of the User Equipments (UEs) and the metric to be optimized and, in some cases, cannot be determined analytically. In order to solve the AP placement problem, global optimization techniques can be employed, as it is the case of the Particle Swarm Optimization (PSO) technique used in this work considering various scenarios with different number of APs, UE distributions, and optimization metrics. The obtained results shown that PSO can be a very suitable tool effectively for the planning of CoMP systems.
\end{abstract}

Keywords-Coordinated Multi-Point, Antenna Port and Particle Swarm Optimization.

\section{INTRODUCTION}

CoMP, also termed DAS, has emerged in recent years, e.g., in the 3GPP LTE [1], as a promising architecture to enhance the link performance and, consequently, the capacity of wireless communication systems. A CoMP system consists of several APs geographically spread over a coverage area and connected through a fast backhaul to an eNB, which might also have an AP [2]-[8]. CoMP systems decrease the average access distances between APs and UEs and, at the same time, they allow for handling the coverage efficiently, decreasing transmit powers, and/or increasing system capacity.

The actual number and placement of APs depend on the average geographical distribution of UEs over the coverage area and the metric to be optimized. Such a metric can include Quality of Service (QoS) requirements and depends on aspects, like the propagation environment, that must be taken into account during the network planning stage.

This work proposes to employ PSO as a means to quasioptimally place a number of APs over a certain coverage region. As it will be seen later in this work, applying PSO with an adequate optimization metric can improve significantly the spectral efficiency of the system, thus making of PSO a valuable tool during the network planning stage.

The rest of this paper is organized as follows. Section II presents the system model considered in this work including the considered scenario described in Section II-A, the PSO modeling is discussed in Section II-B, and the formulation

This work was supported by the Research and Development Center, Ericsson Telecomunicações S.A., Brazil, under EDB/UFC.22 Technical Cooperation Contract. of the AP placement problem in Section II-C. In Section III, some results on the performance of the proposed algorithm are presented. Finally, some conclusions are drawn in Section IV.

\section{System Model ANd AP Placement Problem}

\section{A. General scenario}

We consider the Downlink (DL) of a single CoMP cell with a number $M$ of APs, which are indicated by $m=1,2, \ldots, M$, and are controlled by an eNB. The CoMP system has a total transmission power $P$ and serves a number $J$ of single-antenna UEs, which are indicated by $j=1,2, \ldots, J$.

These $J$ UEs are distributed over the coverage area according to some spatial UE distribution. The average path loss of a UE $j$ distant $d_{j, m}$ kilometers of an AP $m$ is denoted by $P L_{j, m}\left(d_{j, m}\right)$ in $\mathrm{dB}$ and by $p l_{j, m}\left(d_{j, m}\right)$ in linear scale. It is assumed that all the $M$ APs cooperatively transmit to any UEs $j$ using Maximum Ratio Transmission (MRT) [9]. Denoting by $P$ the total transmission power of the cell and by $\sigma^{2}$ the average noise power in the system, the Signal-to-Noise Ratio (SNR) $\gamma_{j}$ of the UE $j$, considering MRT, can be written in linear scale as

$$
\gamma_{j}=\frac{P}{\sigma^{2}} \sum_{m=1}^{M} \frac{1}{p l_{j, m}\left(d_{j, m}\right)} .
$$

The SNR given by (1) will be used by the PSO algorithm.

\section{B. Particle Swarm Optimization (PSO)}

PSO is a technique by Kennedy and Eberhart [10], which is based on a social-psychological metaphor and which has its roots on artificial life and evolutionary computation. PSO often finds application in pattern recognition and image processing problems [11]. The advantages of PSO over some other evolutionary optimization techniques are not only its implementation easiness, but also the reduced number of parameters to adjust [12].

A classical structure for applying PSO consists on creating a population of $N$ particles that adapts by stochastically returning towards successful regions previously found in the search space. The PSO algorithm is iterative and each particle $i$ has:

- a position $\mathbf{P}_{n}$ representing a possible solution of the problem;

- a velocity $\mathbf{V}_{n}$;

- and a previous best position $\mathbf{P}_{n}^{\star}$.

There is also a global best position $\mathbf{P}_{g}^{\star}$, which is the best position among all $\mathbf{P}_{n}^{\star}$ at the moment. These variables can be a matrix, a vector or a scalar, depending on the coding of the 
search space. The efficiency of each particle as solution of the problem is measured by a so-called fitness function $\phi\left(\mathbf{P}_{n}\right)$, which is then used to determine $\mathbf{P}_{n}^{\star}$ and $\mathbf{P}_{g}^{\star}$. The $\mathbf{P}_{n}^{\star}$ and $\mathbf{P}_{g}^{\star}$ values are used iteratively in the PSO algorithm to move towards the global optimum, i.e., the overall best position in the search space. Without loss of generality, the term metric will also be used to denote the fitness function.

At each iteration $k$, the velocity of each particle $n$ is updated using its current velocity $\mathbf{V}_{n}[k]$, position $\mathbf{P}_{n}[k]$, and previous best position $\mathbf{P}_{n}^{\star}[k]$, as well as the global best position $\mathbf{P}_{g}^{\star}[k]$. The new position $\mathbf{P}_{n}[k+1]$ of the particle is obtained simply adding the new velocity to the current position, as explained in the sequel.

An inertia weight $w$ can be used to control the impact of the previous velocity on the current velocity [13]. Typically, $w$ is reduced linearly during the simulation as

$$
w[k]=w_{0}-\frac{k}{K}\left(w_{0}-w_{K}\right),
$$

where $w_{0}$ and $w_{K}$ are the initial and final weights, respectively, and $K$ is the maximum number of iterations. The weights $w_{0}$ and $w_{K}$ are usually set to respect $0 \leq w_{K}<w_{0}$. In this way, a global and a local search are pursued at the beginning and at the end of the optimization process, respectively.

The velocity and position of each particle are updated as

$$
\begin{aligned}
\mathbf{V}_{n}[k+1] & =w[k] \cdot \mathbf{V}_{n}[k] \\
& +c_{1} \cdot \varphi_{1} \cdot\left(\mathbf{P}_{n}^{\star}-\mathbf{P}_{n}[k]\right) \\
& +c_{2} \cdot \varphi_{2} \cdot\left(\mathbf{P}_{g}^{\star}-\mathbf{P}_{n}[k]\right), \text { and } \\
\mathbf{P}_{n}[k+1] & =\mathbf{P}_{n}[k]+\mathbf{V}_{n}[k],
\end{aligned}
$$

where $c_{1}$ and $c_{2}$ are two positive constants inherently used by the PSO algorithm and $\varphi_{1}$ and $\varphi_{2}$ are two random variables uniformly distributed in the interval $[0,1]$. Alternatively, a maximum and/or a minimum value of $\mathbf{V}_{n}$ can be defined in order to prevent too much divergence of the solutions. Table I presents a pseudocode of the PSO algorithm.

\section{TABLE I}

PSEUDOCODE FOR THE PSO ALGORITHM.

1) Randomly initialize $\mathbf{P}_{n}, \mathbf{V}_{n}, \mathbf{P}_{n}^{\star}$ and $\mathbf{P}_{g}^{\star} \forall n \in\{1,2, \ldots, N\}$

2) For each particle $n$

a) If $\phi\left(\mathbf{P}_{n}[k]\right) \lessgtr \phi\left(\mathbf{P}_{n}^{\star}\right)$, set $\mathbf{P}_{n}^{\star}=\mathbf{P}_{n}[k]$

b) If $\phi\left(\mathbf{P}_{n}[k]\right) \lessgtr \phi\left(\mathbf{P}_{g}^{\star}\right)$, set $\mathbf{P}_{g}^{\star}=\mathbf{P}_{n}[k]$

3) For $k=1$ to $K$

a) Update $w[k]$ using (2)

b) For each particle $n$

i) Update the particle velocity $\mathbf{V}_{n}$ using (3a)

ii) Update the particle position $\mathbf{P}_{n}$ using (3b)

iii) If $\phi\left(\mathbf{P}_{n}[k]\right) \lessgtr \phi\left(\mathbf{P}_{n}^{\star}\right)$, set $\mathbf{P}_{n}^{\star}=\mathbf{P}_{n}[k]$

c) For each particle $n$

$$
\text { i) If } \phi\left(\mathbf{P}_{n}[k]\right) \lessgtr \phi\left(\mathbf{P}_{g}^{\star}\right) \text {, set } \mathbf{P}_{g}^{\star}=\mathbf{P}_{n}[k]
$$

The operator $\lessgtr$ is generally used for minimization and maximization problems. If the desired problem is a minimization one, $\lessgtr$ must be replaced by $<$. Otherwise, $\lessgtr$ must be replaced by $>$.

\section{APs Placement Problem}

Determining an optimal APs placement can be easy or hard to solve depending on three main characteristics:

- the UE distribution over the coverage area;

- the number $M$ of APs;

- the metric $\phi(\cdot)$ to be optimized.

As a simple example, consider a uniform distribution of UEs over a circular sector, defined by its radius $R$ and its an angle $\Theta$, and one AP to be placed. In this scenario, the problem of minimizing the average access distance $d(\cdot)$ of the UEs can be solved analytically. The access distance of a UE is defined as the distance between this UE and the closest AP to it. The solution of this problem can be written as

$$
\left[r_{A P}^{*}, \theta_{A P}^{*}\right]=\arg \min \left[d\left(r_{A P}, \theta_{A P}\right)\right],
$$

where $r_{A P}$ and $\theta_{A P}$ are, respectively, the radius and the angle of AP position. Instead of minimize $d(\cdot)$, we will minimize $d^{2}(\cdot)$, which will produce the same result. Considering all the points of the desired area, represented by the polar coordinates $r$ and $\theta, d^{2}(\cdot)$ is given by

$$
\begin{array}{r}
d^{2}\left(r_{A P}, \theta_{A P}\right)=\int_{0}^{\Theta} \int_{0}^{R}\left[r_{A P}^{2}-2 r_{A P} \cdot r .\right. \\
\left.\cos \left(\theta-\theta_{A P}\right)+r^{2}\right] r \cdot d r \cdot d \theta .
\end{array}
$$

Solving the integrals, we obtain

$$
\begin{aligned}
d^{2}\left(r_{A P}, \theta_{A P}\right) & =r_{A P}^{2} \cdot \frac{R^{2}}{2} \cdot \Theta-2 r_{A P} \cdot \frac{R^{3}}{3} . \\
& {\left[\sin \left(\Theta-\theta_{A P}\right)+\sin \left(\theta_{A P}\right)\right]+\Theta \frac{R^{4}}{4} . }
\end{aligned}
$$

This function is continuous and its derivatives of first and second order exists and are continuous too. In order to determine a minimum point, the gradient operator have to be used. The gradient vector is given by (7).

$$
\nabla d^{2}\left(r_{A P}, \theta_{A P}\right)=\left[\begin{array}{c}
\frac{\partial d^{2}\left(r_{A P}, \theta_{A P}\right)}{\partial r_{A P}} \\
\frac{1}{r_{A P}} \cdot \frac{\partial d^{2}\left(r_{A P}, \theta_{A P}\right)}{\partial \theta_{A P}}
\end{array}\right]
$$

Equaling the two components to zero, we obtain a solution:

$$
\begin{aligned}
& \theta_{A P}=\frac{\Theta}{2}, \text { and } \\
& r_{A P}=\frac{4 R}{3 \Theta} \cdot \sin \left(\frac{\Theta}{2}\right),
\end{aligned}
$$

which can be a minimum, a maximum or a cell point of the function $d^{2}(\cdot)$. To determine what this point is, the Hessian matrix $\mathbf{H}\left(d^{2}\left(r_{A P}, \theta_{A P}\right)\right)$, which will be denoted by $\mathbf{H}\left(d^{2}(\cdot)\right)$, have to be calculated according to $(9 \mathrm{a})$.

$$
\begin{aligned}
& \mathbf{H}\left(d^{2}(\cdot)\right)=\left[\begin{array}{cc}
\frac{\partial^{2} d^{2}(\cdot)}{\partial r_{A P}^{2}} & \frac{1}{r_{A P}} \frac{\partial^{2} d^{2}(\cdot)}{\partial r_{A P} \partial \theta_{A P}} \\
\frac{1}{r_{A P}} \frac{\partial^{2} d^{2}(\cdot)}{\partial \theta_{A P} \partial r_{A P}} & \frac{1}{r_{A P}^{2}} \frac{\partial^{2} d^{2}(\cdot)}{\partial \theta_{A P}^{2}}
\end{array}\right] \\
& \mathbf{H}\left(d^{2}(\cdot)\right)=\left[\begin{array}{cc}
R^{2} \Theta^{4} & 0 \\
0 & R^{2} \Theta^{4}
\end{array}\right]
\end{aligned}
$$

This matrix is positive defined [14] and the found solution is, therefore, a minimum point of $d^{2}(\cdot)$. Note that if $\Theta=2 \pi$, 
which will corresponds to a circular area, the AP has to be placed at the center of the area.

In some cases, an analytical approach, such as the previously presented one, is infeasible. In these cases, PSO can comes in play to solve the problem numerically. Consider $M$ APs with positions described by Cartesian coordinates. Thus, we describe a generic solution of the problem as

$$
\mathbf{P}_{n}[k]=\left[\begin{array}{llll}
x_{n}^{(1)}[k] & x_{n}^{(2)}[k] & \ldots & x_{n}^{(M)}[k] \\
y_{n}^{(1)}[k] & y_{n}^{(2)}[k] & \ldots & y_{n}^{(M)}[k]
\end{array}\right]
$$

where $x_{n}^{(m)}[k]$ and $y_{n}^{(m)}[k]$ are respectively the horizontal and vertical coordinates of the $m^{t h}$ AP of the $n^{t h}$ particle at iteration $k . \mathbf{V}_{n}, \mathbf{P}_{n}^{\star}$ and $\mathbf{P}_{g}^{\star}$ have the same dimensions of $\mathbf{P}_{n}$.

The PSO has to place the APs in the coverage area as to optimize the fitness of the best global position $\mathbf{P}_{g}^{\star}$. Thus, it is necessary to define a suitable fitness function $\phi(\cdot)$. Some possible fitness functions or, equivalently, optimization objectives, are:

- minimize the average access distance among the UEs and APs;

- maximize the lowest SNR among all UEs;

- maximize the average SNR of the UEs;

- maximize the average Signal to Interference plus Noise Ratio (SINR) of the UEs.

According to the adopted fitness function, the PSO algorithm might obtain different results, i.e., different topologies for the set of APs. This work considers two different fitness functions: average access distance and lowest SNR of the UEs, represented, respectively, by $\phi_{1}$ and $\phi_{2}$. These two metrics will lead a minimization and a maximization problem respectively. Mathematically these problems can be formulated as follows:

$$
\begin{aligned}
& \text { Minimize: } \phi_{1}=\frac{1}{J} \sum_{j=1}^{J} d_{j} \text {, and } \\
& \text { Maximize: } \phi_{2}=\min \left(\left[\gamma_{1}, \gamma_{2}, \cdots, \gamma_{J}\right]\right),
\end{aligned}
$$

where $d_{j}$ denotes the access distance of the UE $j$. The obtained solutions for these two problems are termed Access Distance Minimized (ADM) and Lowest SNR Maximized (LSM) respectively.

\section{RESULTS}

In order to determine an adequate topology for a set of $M$ APs, the PSO algorithm of Table I is applied here in a scenario consisting of 20000 UEs spread over the coverage area according to uniform and non-uniform distributions, considering different fitness functions, and considering a variable number of APs. The average path loss of a UE $j$ distant $d_{j, m}$ kilometers of an AP $m$ has been modeled according to the Okumura-Hata model of [15] and is given by

$$
P L_{j, m}\left(d_{j, m}\right)=128.1+37.6 \log _{10}\left(d_{j, m}\right) \text { in } \mathrm{dB} \text {. }
$$

The main parameters considered in the simulations are summarized in Table II. The constants $c_{1}$ and $c_{2}$ are set to be 2 in order to keep the stochastic factor's mean equals to 1 [10]. A convergence test was made ranging $w_{K}$. The values of $0,0.6$ and 1.2 was analysed in a scenario consisting of 4 APs, UEs uniformly distributed and $\phi_{1}$ as the metric to be optimized. Figure III shows the average access distance of the UEs versus the number of iterations of PSO. As we can see, when $w_{K}$ is set to be 0, PSO algorithm has a faster convergence. Therefore, $w_{0}=1.2$ and $w_{K}=0$ were used, as proposed in [16].

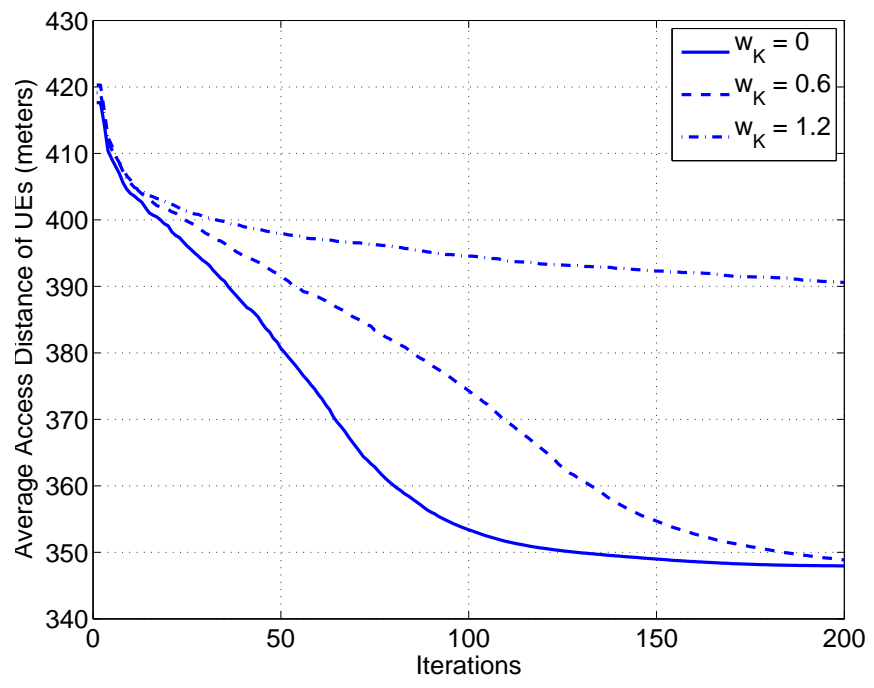

Fig. 1. Average access distance versus number of iterations of PSO for

\begin{tabular}{|c|c|c|}
\hline Parameter & Symbol & Value \\
\hline \multicolumn{3}{|c|}{ System } \\
\hline Total transmit power & $P$ & $21.25 \mathrm{dBm}$ \\
\hline Coverage radius & $R$ & $1 \mathrm{~km}$ \\
\hline Noise power & $\sigma^{2}$ & $-123.24 \mathrm{dBm}$ \\
\hline Number of UEs & $J$ & 20000 \\
\hline \multicolumn{3}{|c|}{$\overline{\text { PSO }}$} \\
\hline Number of particles & $\bar{N}$ & $\overline{200}$ \\
\hline Number of iterations & $K$ & 100 \\
\hline Initial inertia weight & $w_{0}$ & 1.2 \\
\hline Final inertia weight & $w_{K}$ & 0 \\
\hline Constants & $c_{1}, c_{2}$ & 2 \\
\hline
\end{tabular}
different parameters.

TABLE II

SIMULATION PARAMETERS.

The APs placement problem has three main characteristics: number $M$ of APs, average distribution of the UEs over the coverege area and the metric to be optimized. In the following, these characteristics are varied and, then, the PSO is used to determine the optimal placement of the APs. This procedure will show that PSO can adapts its solution to any APs placement problem. First, we fix the number of APs to $M=4$ and analyze the solution for the AP placement problem found by the PSO algorithm for three different UEs distributions: uniform, hot-spot and centralized distributions.

Considering a uniform distribution, all points within the coverage area have the same density of UEs. The hot-spot distribution considers a smaller area, within the total area of 


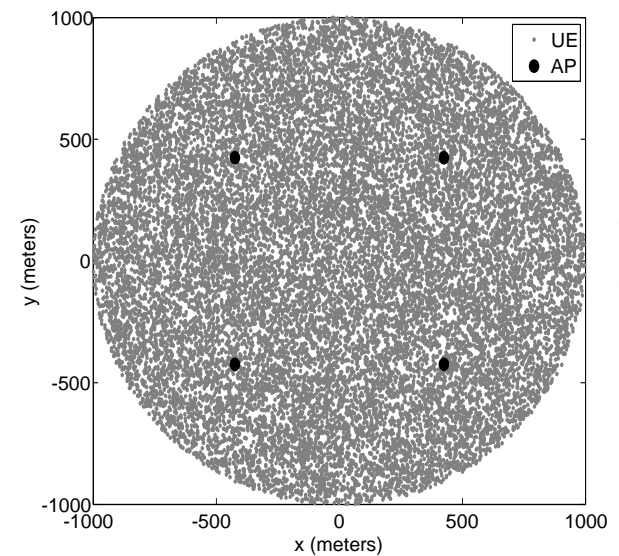

(a) Uniformly distributed UEs.

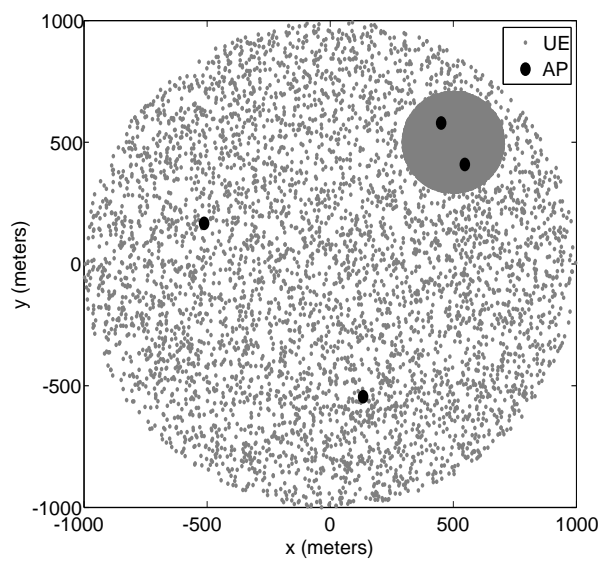

(b) $75 \%$ of the UEs at the hot spot.

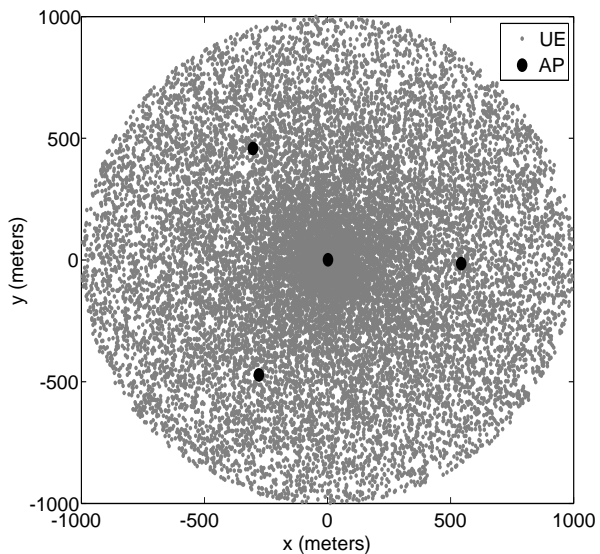

(c) Centralized distribution.

Fig. 2. Topologies for different UEs distributions.

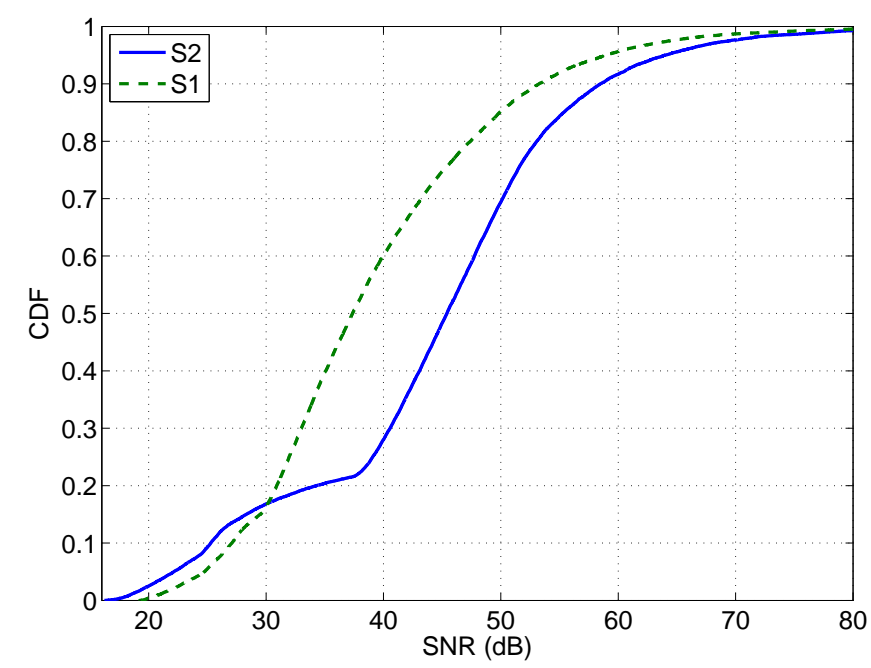

(a) Hot-Spot distribution.

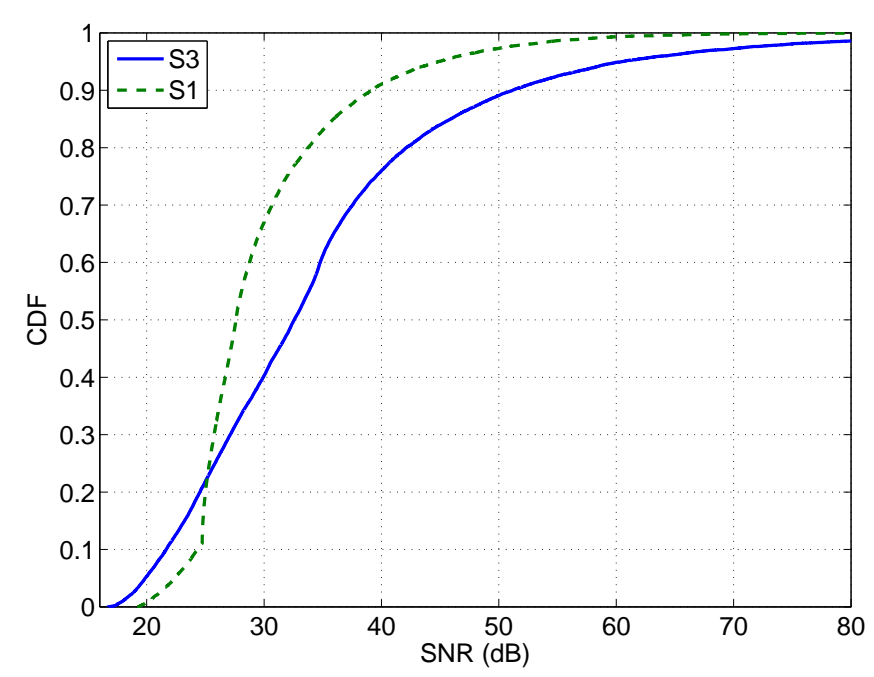

(b) Centralized distribution.

Fig. 3. CDF for different UEs distribution and different solutions. the cell, with higher density of UEs. The radius of this region was set to be $R_{h s}=50 \mathrm{~m}$. The UEs distribution inside the hot-spot is uniform. Finally, the centralized distribution have a greater density of UEs at the center of the coverage area and this density decreases with the growth of the distance between a desired point and the center of the coverage area. The obtained AP topologies are shown in Figures 2(a), 2(b) and 2(c).

Considering a uniform distribution, $M=4$ and the average access distance as the metric to be optimized, the APs placement problem can be solved analytically. To solve this problem, we need to split the coverage area in four equal circular sectors. The new problem is to positionate one AP in each sector in order to minimize the average access distance of the UEs. This new problem corresponds to the problem solved analytically in Section II-C, with $\Theta=\pi / 2$. The solution for this problem, according to (8), is $\theta_{A P}=\pi / 2$ and $r_{A P}=0.6 \mathrm{~km}$. PSO obtains the same solution as we can see in Figure 2(a). Let this solution be detoned as $S_{1}$.

However, the other two problems are not as simple as the problem aforementioned. Figures 2(b) and 2(c) show the obtained topologies for these problems by PSO. Suppose that the PSO tool is not available and that is required that the problems of hot-spot and centralized distribution have to be solved. In this case, considering that an analytical approach is not feasibe, we could apply the solution $S_{1}$ to these two problems. The PSO solution for these two problems are named, respectively, $S_{2}$ and $S_{3}$. Figure 3(a) shows the Cumulative Distribution Function (CDF) of the SNRs of the UEs spreaded over the coverage area for $S_{1}$ and $S_{2}$. Solution $S_{2}$ provided an average SNR of $45 \mathrm{~dB}$ versus $37.3 \mathrm{~dB}$ of solution $S_{1}$, that is, a gain of $7.7 \mathrm{~dB}$. The same analysis can be done in Figure 3(b). Solution $S_{3}$ provided an average SNR of $32.6 \mathrm{~dB}$ versus $27.6 \mathrm{~dB}$ of solution $S_{1}$. A gain of $5 \mathrm{~dB}$. The crossing between the curves in Figures 3(a) and 3(b) occurs due to the better coverage of $S_{1}$ at the edge of the cell. This is because $S_{1}$ places the APs closer to the cell edge than $S_{2}$ and $S_{3}$. However, in both situations, most of the UEs are not close to the cell edge.

In the following, we consider a uniform distribution of the 
UEs and two different optimization objectives i.e., different metrics: $\phi_{1}$ and $\phi_{2}$. These two objectives were analyzed considering $M=4,5$, and 7 APs. Two different cases are considered: one in which no AP is forced to lie in the center of the coverage area and another in which this occurs. This latter case resembles current cellular networks and it is of interest here because it might be seen as a system upgrade phase, i.e., a system APs deployed in a previous phase must have their positions preserved in the new extended topology. The description of the obtained AP topologies is shown in Table III.

TABLE III

RADIUS (IN KM) OF AP DISTRIBUTION AROUND THE CENTER OF THE COVERAGE AREA.

\begin{tabular}{|c|c|c|c|c|c|c|}
\hline \multicolumn{6}{|c|}{ Number of APs } & \multirow{3}{*}{$\begin{array}{l}\text { One AP fixed at } \\
\text { the center of the } \\
\text { coverage area }\end{array}$} \\
\hline \multicolumn{3}{|c|}{ ADM } & \multicolumn{3}{|c|}{ LSM } & \\
\hline $\bar{M}=4$ & $M=5$ & $\mathbf{M}=7$ & $\mathrm{M}=4$ & $\mathbf{M}=\mathbf{5}$ & $\mathrm{M}=7$ & \\
\hline 0.60 & 0.62 & $\overline{-}$ & 0.70 & 0.74 & 0.67 & No \\
\hline 0.62 & 0.66 & 0.69 & 0.46 & 0.70 & 0.86 & Yes \\
\hline
\end{tabular}

For all the obtained topologies, adjacent APs are separated angularly of $\alpha=2 \pi / M^{\prime}$, where $M^{\prime}$ is the total number of APs $M$ minus the number of APs at the center of the CoMP cell, if any. For a visualization example, see Figure 2(a), in which the radius of the APs distributed around the center of the coverage area is $0.60 \mathrm{~km}$ and $\alpha=\pi / 2$.

Observing the results presented in Table III, it can be noted that although no AP is forced to lie in the center of the coverage area in the first case, this situation occurs when considering the ADM metric and $M=7$ APs.

In order to compare the ADM and LSM topology solutions and give an idea of the coverage situation in each case, the CDFs of the SNR of the UEs for $M=4,5$, and 7 APs are presented in Figure 4. The SNR of each UE is calculated according to (1).

We can verify the SNR levels perceived in the CoMP cell by considering, e.g., the $10^{\text {th }}$ percentile of the CDFs presented in Figure 4, i.e., the SNR level perceived by $90 \%$ of the UEs. From these results, the adequacy of each metric as a means to quantify the obtained coverage can be compared. Table IV summarizes these values for each metric and topology.

TABLE IV

$10^{\mathrm{TH}}$ PERCENTILE OF SNR (IN DB) FOR EACH AP DISTRIBUTION.

\begin{tabular}{|c|c|c|c|c|c|c|}
\hline \multicolumn{6}{|c|}{ Number of APs } & \multirow{3}{*}{$\begin{array}{l}\text { One AP fixed at } \\
\text { the center of the } \\
\text { coverage area }\end{array}$} \\
\hline \multicolumn{3}{|c|}{ ADM } & \multicolumn{3}{|c|}{ LSM } & \\
\hline $\mathrm{M}=4$ & $\mathrm{M}=5$ & $\mathrm{M}=7$ & $\mathrm{M}=4$ & $\mathrm{M}=5$ & $\mathrm{M}=7$ & \\
\hline 22.5 & $\overline{24.0}$ & - & $\overline{22.6}$ & 23.1 & $\overline{24.9}$ & " No \\
\hline 19.9 & 22.2 & 25.5 & 19.6 & 22.3 & 24.8 & $\overline{\text { Yes }}$ \\
\hline
\end{tabular}

It can be noted that the ADM solution was better in the cases of 5 and 7 APs. The solutions that place one AP at center were worse than the other ones, except for the case of ADM with 7 APs. In this case, the solution with one AP at center is the optimum one. The results show that, although the LSM maximizes the lowest SNR (as we can see in Figures 4(a), 4(b) and 4(c)), it does not ensure the best coverage. Moreover, results presented at Table IV show that the coverage is improved with the growth of $M$.

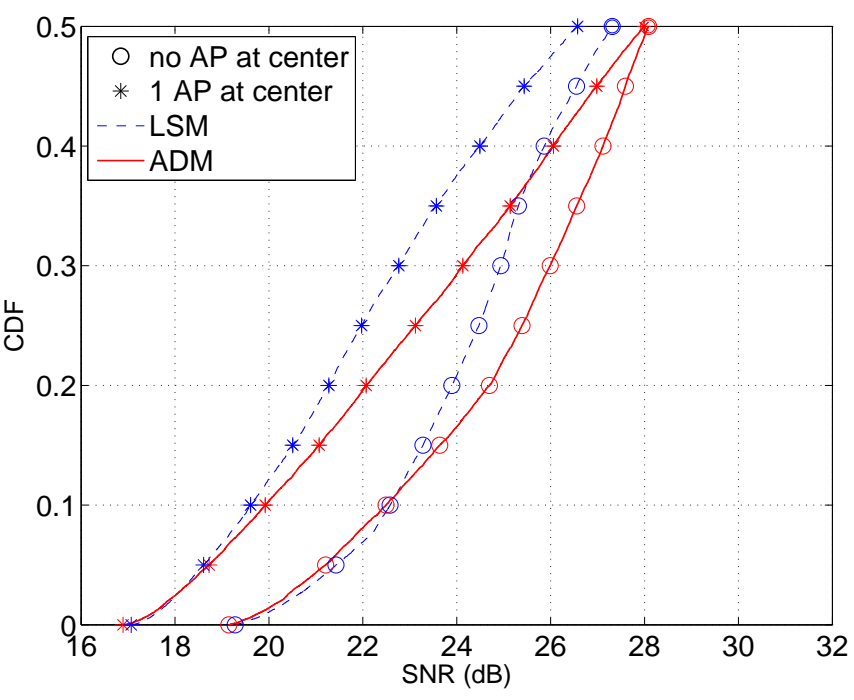

(a) $M=4 \mathrm{APs}$

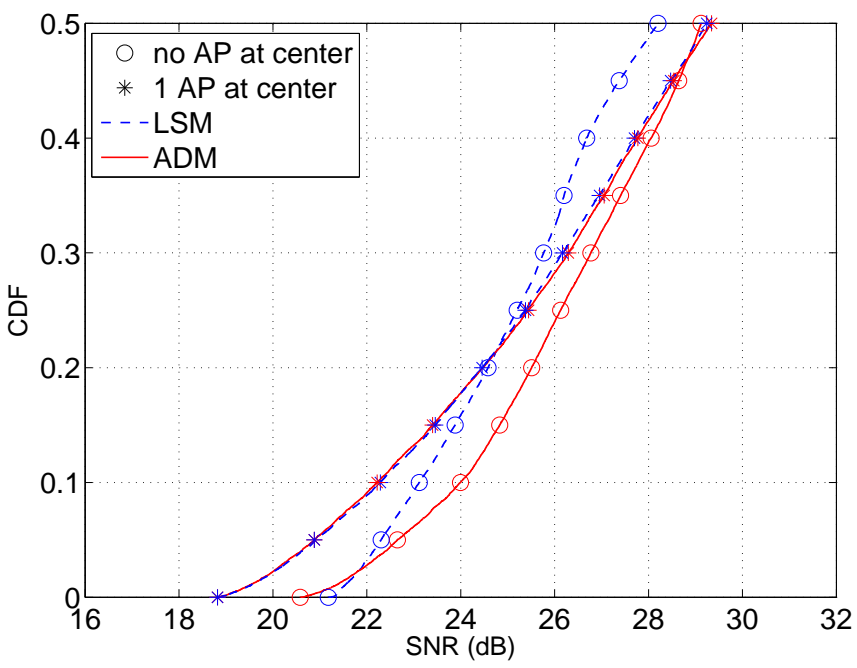

(b) $M=5 \mathrm{APs}$

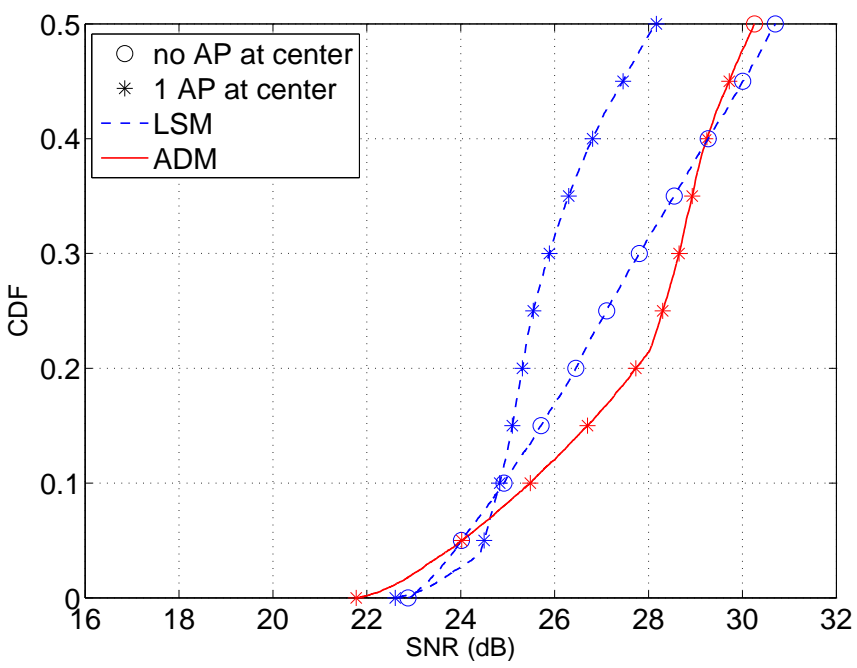

(c) $M=7 \mathrm{APs}$

Fig. 4. Cumulative Distribution Function of UEs' SNRs. 


\section{CONCLUSIONS}

This work presented an important application of PSO. It was shown that a PSO solution for the APs placement problem can bring a huge gain of spectral efficiency to a CoMP system. PSO can find the same solution of a problem solved analytically and can find a solution for problems infeasible analytically. The main parameters of the APs placement problem were varied and the results showed that PSO can adapts its solution to any APs placement problem. The technique presented in this work have shown consistent results even in complex scenarios. Due to this, it could be used as an efficient tool at the network planning stage.

\section{REFERENCES}

[1] S. D. Parkvall, "Lte-advanced - evolving lte towards imt-advanced," in Vehicular Technology Conference, 2008.

[2] A. A. M. Saleh, A. J. Rustako Jr., and R. S. Roman, "Distributed antennas for indoor radio communications," IEEE Transactions on Communications, vol. 35, pp. 1245-1251, 1987.

[3] M. V. Clark, T. M. Willis, L. J. Greenstein, A. J. Rustako Jr., V. Ercegt, and R. S. Roman, "Distributed versus centralized antenna arrays in broadband wireless networks," in Proceedings of the IEEE Vehicular Technology Conference (VTC), 2001.

[4] W. Choi and J. G. Andrews, "Downlink performance and capacity of distributed antenna systems in a multicell environment," IEEE Transactions on Wireless Communications, vol. 6, no. 1, pp. 69-73, Jul1 2007.

[5] J. Zhang and J. G. Andrews, "Cellular communication with randomly placed distributed antennas," in Proceedings of the IEEE Global Telecommunications Conference, 2007.
[6] S. Zhou, M. Zhao, X. Xu, J. Wang, and Y. Yao, "Distributed wireless communication system: A new architecture for future public wireless access," IEEE Communications Magazine, pp. 108-113, Jul3 2003.

[7] S. Xinghua, H. Zhiqiang, N. Kai, and W. Weiling, "A hierarchical resource allocation for OFDMA distributed wireless communication systems," in Proceedings of the IEEE Global Telecommunications Conference, 2007.

[8] H. Hu, Y. Zhang, and J. Luo, Eds., Distributed antenna systems: open architecture for future wireless communications, 1st ed., ser. Wireless networks and mobile communications. Auerbach, Jul 2007.

[9] T. K. Y. Lo, "Maximum ratio transmission," IEEE Transactions on Communications, vol. 47, No. 10, pp. 1458-1461, October, 1999.

[10] J. Kennedy and R. Eberhart, "Particle swarm optimization," in Proceedings of the 1995 IEEE International Conference on Neural Networks, Vol. 4, 1995, pp. 1942-1948.

[11] M. G. H. Omran, "Particle swarm optimization methods for pattern recognition and image processing," Ph.D. dissertation, Faculty of Engineering, Built Envi. and IT, University of Pretoria, 2004.

[12] P. Angeline, "Evolutionary optimization versus particle swarm optimization: Philosophy and performance differences," Evolutionary Programming, Lecture Notes in Computer Science, Springer, vol. 1447, pp. 601610, 1998.

[13] M. Inthachot and S. Supratid, "A multi-subpopulation particle swarm optimization: A hybrid intelligent computing for function optimization," 2007.

[14] C. M. S. Mokhtar S. Bazaraa, Hanif D. Sherali, Nonlinear Programming - Theory and Algorithms, 2nd ed., 1993.

[15] UMTS, "Selection procedures for the choice of radio transmission technologies of the UMTS," UMTS, Tech. Rep., 1998.

[16] R. C. Eberhart and Y. Shi, "Comparison between genetic algorithms and particle swarm optimization," Lecture Notes in Computer Science, vol. 1447, pp. 611-616, 1998. 